\title{
ETHICAL AND ORGANIZATIONAL DILEMMAS RELATED TO THE TREATMENT OF COVID- 19 PATIENTS
}

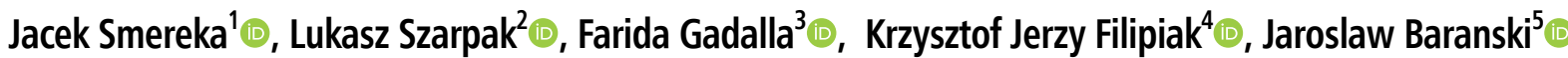 \\ ${ }^{1}$ Department of Emergency Medical Service and Laboratory of Experimental Medicine and Innovative Technology, \\ Wroclaw University of Medicine, Wroclaw, Poland \\ ${ }^{2}$ Lazarski University, Warsaw, Poland \\ ${ }^{3}$ Department of Anesthesiology, Weill Cornell Medicine, New York, USA \\ ${ }^{4}$ First Chair and Department of Cardiology, Medical University of Warsaw, Poland \\ ${ }^{5}$ Department of Humanistic Sciences in Medicine, Wroclaw Medical University, Wroclaw, Poland
}

KEY WORDS: ethics, COVID-19, intensive care, public health

Disaster Emerg Med J 2020; 5(3): 121-123

The current COVID-19 epidemic and SARS-CoV-2 infections pose a huge challenge to health systems in many countries, even those with the most developed infrastructure, capacity, and equipment. For less developed countries, the COVID-19 epidemic represents a tragedy on an unprecedented scale, where inferior health systems and depressed economies mean that large parts of the population on the verge of poverty are deprived of adequate medical care [1]. Lack of work and diminished livelihoods compound the already uncontrolled development of the epidemic. While in developed countries, measures including quarantine, isolation, 'stay home' messaging and the use of masks by healthy people can reduce the number of cases, it is already clear that these health care systems are also on the verge of collapse [1].

The current situation, which is linked to the lack of availability of medical equipment, including ventilators and personal protective equipment (PPE), challenges cooperation between countries [2]. Italy's lack of sufficient support in its heroic fight against the pandemic is the best example of this. This problem may also affect hospitals in countries or regions that compete for medical equipment and personnel. Because small hospitals often have significantly smaller resources (both in staffing and supplies) compared to large hospitals, their inability to function normally will greatly affect local communities [3]. The current COVID-19 pandemic especially requires transparency of decision-making and engagement of stakeholders and its impact on the public perception of fairness.

The lack of availability of advanced life-saving methods, such as ventilation and intensive care makes it necessary to segregate patients who will be provided with high-quality medical assistance [4]. Such situations are usually single events limited in time. Each intensive care unit makes decisions related to ICU patient qualification in case of an imbalance between the number of patients and the availability of ICU beds. However, in the case of COVID-19, the problem of making choices over many weeks or even months arises. In addition, the health care system may have to make decisions about a serious reduction in the number of patients treated in the ICU.

One of the criteria used in some countries is the age of the patient. Otherwise, a 60 -year-old patient with coexisting diseases would almost certainly have the possibility of ICU treatment, which is currently limited in many countries. It should be remembered that decisions about limitation are made not automatically by the healthcare or hospital systems or committees, but by individual persons - physicians, often after consultation with other specialists. This is a situation which we have not experienced before on such a scale. It raises a number of ethical and, in the long run, perhaps legal issues. Some physicians who have to make such decisions may also require psychological support and debriefing. 
A separate issue that is addressed is non-medical indications - the social usefulness suggested in some countries. This raises further ethical doubts as to the order in which assistance should be provided and which patients should be qualified for ICU treatment, whether the disabled have less right to life, whether their education or profession may be a criterion for qualification for intensive treatment. All these utilitarian (generally, social) criteria are intuitive, unjustified, and subjective. Therefore, this problem requires recourse to normative ethics, as medical science alone may be insufficient to justify such medical decisions $[5,6]$.

The application of the age and 'social usefulness' (e.g. occupation, proposed in some countries) criteria in the access to the ventilator in a situation of COVID-19 pandemic and overloaded health care system raise fundamental ethical concerns. These violate the principle of equity in access to medical services and place an enormous burden on the decision-makers' morals. Earlier attempts were made to formulate other criteria, taking into account the experience of the SARS pandemic, considering them fairer than the age criterion, e.g. physicians may employ a lottery process [7] or currently arguing for a limited time to use a ventilator for a patient with acute respiratory failure [8], giving everyone a chance to survive. The Swiss Society of Intensive Care Medicine (SSIM) and the Swiss Academy of Medical Sciences (SAMS) have developed guidelines that avoid the use of the age criterion, considering that such a criterion violates the prohibition of discrimination, and based on medical considerations as to which patients will benefit most from intensive care. Clear and, as far as possible, simple guidelines for decision-making are therefore needed, since the state of the pandemic and overburdened health systems provoke strong paternalism with the risk of arbitrary decisions by physicians and hospital management [6].

These are problems that require solutions. It should be remembered that after the end of the pandemic there will come a time to analyze the decisions that were made. It may turn out that the system of values on which the modern world is based will have to be updated, that the principle of equality does not always apply. However, it seems that a pandemic does not so much re-evaluate our system of values, but puts it to the test. Are we able, in a post-pandemic world, to accept behavior that refuses to care for the elderly or the very sick, which, contradicts what European culture has recognized as a moral obligation to others? What are the costs of the arbitrary justification of medical decisions concerning patients with coexisting diseases or elderly patients who have been refused life-saving assistance? Lack of trust in health services, physicians and nurses, seems to be the smallest problem.

At a time of universal shortages of personal protective equipment (PPE) for medical personnel in most countries, consideration should be given to whether the exposure of a physician, nurse, or paramedic is ethically acceptable. In the case of a procedure requiring an immediate attention, how much time should be spent donning and checking protective clothing? Is any delay that endangers the patient on one hand, and the staff on the other, ethically justified? Most often, codes of ethics and bioethical declarations do not mention the obligations of medical personnel in the event of a pandemic [9]. In the medical education system, there is no risk of practicing the profession by sacrificing one's own life for the benefit of the patient [4].

Another important aspect of the current epidemic is timely access to medical care. Some patients will deteriorate quickly or lose their lives due to obstacles in accessing the health care system - physical means of access (transportation), inadequate staffing, or even delays due to the fear of infection in a hospital [10]. Temporary restrictions on the activities of some health care units, including entire wards, or even the transformation of hospitals into infectious ones, may result in delays or complete prevention of care. There is no doubt the COVID-19 pandemic will increase medical needs, but after the pandemic, will we be able to finance the increase? What criteria for access to medical services must then be formulated in the event of a deficit of benefits and personnel caused by the pandemic?

Prior Presentations: None.

Funding Sources/Disclosures: None.

Acknowledgments: None.

\section{REFERENCES}

1. Litewka SG, Heitman E. Latin American healthcare systems in times of pandemic. Dev World Bioeth. 2020; 20(2): 69-73, doi: 10.1111/ dewb.12262, indexed in Pubmed: 32282974.

2. Hilsenrath PE. Ethics and Economic Growth in the Age of COVID-19: What Is a Just Society to Do? J Rural Health. 2020 [Epub ahead of print], doi: 10.1111/jrh.12434, indexed in Pubmed: 32246515. 
3. Patel L, Elliott A, Storlie E, et al. Ethical and Legal Challenges During the COVID-19 Pandemic: Are We Thinking About Rural Hospitals? J Rural Health. 2020 [Epub ahead of print], doi: 10.1111/jrh.12447, indexed in Pubmed: 32282953.

4. Mannelli C. Whose life to save? Scarce resources allocation in the COVID-19 outbreak. J Med Ethics. 2020; 46(6): 364-366, doi: 10.1136/ medethics-2020-106227, indexed in Pubmed: 32277018.

5. Kotalik J. Preparing for an influenza pandemic: ethical issues. Bioethics. 2005; 19(4): 422-431, doi: 10.1111/j.1467-8519.2005.00453.x, indexed in Pubmed: 16222857.

6. Thompson AK, Faith K, Gibson JL, et al. Pandemic influenza preparedness: an ethical framework to guide decision-making. BMC Med Ethics. 2006; 7: E12, doi: 10.1186/1472-6939-7-12, indexed in Pubmed: 17144926.
7. Bagenstos S. May Hospitals Withhold Ventilators from COVID-19 Patients with Pre-Existing Disabilities? Notes on the Law and Ethics of Disability-Based Medical Rationing. SSRN Electronic Journal. , doi: 10.2139/ssrn.3559926.

8. White DB, Lo B. A Framework for Rationing Ventilators and Critical Care Beds During the COVID-19 Pandemic. JAMA. 2020 [Epub ahead of print], doi: 10.1001/jama.2020.5046, indexed in Pubmed: 32219367.

9. Ruderman C, Tracy CS, Bensimon CM, et al. On pandemics and the duty to care: whose duty? who cares? BMC Med Ethics. 2006; 7: E5, doi: 10.1186/1472-6939-7-5, indexed in Pubmed: 16626488.

10. Kramer JB, Brown DE, Kopar PK. Ethics in the Time of Coronavirus: Recommendations in the COVID-19 Pandemic. J Am Coll Surg. 2020; 230(6): 1114-1118, doi: 10.1016/j.jamcollsurg.2020.04.004, indexed in Pubmed: 32278728. 